\title{
Full charge-density calculation of the surface energy of metals
}

\author{
Vitos, Levente; Kollár, J..; Skriver, Hans Lomholt
}

Published in:

Physical Review B

Link to article, DOI:

10.1103/PhysRevB.49.16694

Publication date:

1994

Document Version

Publisher's PDF, also known as Version of record

Link back to DTU Orbit

Citation (APA):

Vitos, L., Kollár, J., \& Skriver, H. L. (1994). Full charge-density calculation of the surface energy of metals. Physical Review B, 49(23), 16694-16701. https://doi.org/10.1103/PhysRevB.49.16694

\section{General rights}

Copyright and moral rights for the publications made accessible in the public portal are retained by the authors and/or other copyright owners and it is a condition of accessing publications that users recognise and abide by the legal requirements associated with these rights.

- Users may download and print one copy of any publication from the public portal for the purpose of private study or research.

- You may not further distribute the material or use it for any profit-making activity or commercial gain

- You may freely distribute the URL identifying the publication in the public portal

If you believe that this document breaches copyright please contact us providing details, and we will remove access to the work immediately and investigate your claim 


\title{
Full charge-density calculation of the surface energy of metals
}

\author{
L. Vitos and J. Kollár \\ Research Institute for Solid State Physics, P.O. Box 49, H-1525 Budapest, Hungary \\ H. L. Skriver \\ Center for Atomic-scale Materials Physics and Physics Department, \\ Technical University of Denmark, DK-2800 Lyngby, Denmark
}

(Received 25 January 1994)

\begin{abstract}
We have calculated the surface energy and the work function of the $4 d$ metals by means of an energy functional based on a self-consistent, spherically symmetric atomic-sphere potential. In this approach the kinetic energy is calculated completely within the atomic-sphere approximation (ASA) by means of a spherically symmetrized charge density, while the Coulomb and exchangecorrelation contributions are calculated by means of the complete, nonspherically symmetric charge density within nonoverlapping, space-filling Wigner-Seitz cells. The functional is used to assess the convergence and the accuracy of the linear-muffin-tin-orbitals (LMTO) method and the ASA in surface calculations. We find that the full charge-density functional improves the agreement with recent full-potential LMTO calculations to a level where the average deviation in surface energy over the $4 d$ series is down to $10 \%$.
\end{abstract}

\section{INTRODUCTION}

During the last years one has seen a number of systematic studies of the surface energies and work functions of simple and transition metals by means of $a b$ initio total energy calculations. These include the fullpotential linear-muffin-tin-orbitals (LMTO) slab calculations by Methfessel et $a l^{1}{ }^{1}$ and the Green's function calculations by Skriver and Rosengaard. ${ }^{2-4}$ In the comparison between the two sets of calculations for the $4 d$ transition series it was concluded that the Green's function technique based on the atomic-sphere approximation (ASA) gave surface energies in excellent agreement with the full-potential results, especially for the late elements in the series. ${ }^{4}$

It is natural to extend the LMTO surface calculations to the actinide-metal series by including $f$ states in the basis set. However, when the Green's function technique was applied to the light actinides, it was found that the surface energy of the two first actinides, Ac and $\mathrm{Th}$, were slightly negative. ${ }^{5}$ Similarly, when $f$ states were included in surface calculations for the $4 d$ series the excellent agreement between the ASA based technique and the full-potential calculations disappeared, as may be seen in Fig. 1 where we compare calculated and experimentally derived surface energies. ${ }^{6}$ The fact that the failure was particularly pronounced at the beginning of a $d$ or $f$ transition series, where the charge density at the boundary of the Wigner-Seitz sphere is relatively large, pointed to an inadequate evaluation of the Coulomb and exchangecorrelation contributions to the total energy.

An essential aspect of the LMTO Green's function technique which is based on the work of Andersen and co-workers $^{7-13}$ is the ability, within the ASA and in the tight-binding representation, to generate the Green's function matrices for a real, two-dimensional interface by a simple and efficient procedure. In addition, the ASA allows an efficient and accurate determination of the kinetic energy and, according to the agreement with the fullpotential calculations, apparently also of the Coulomb and exchange-correlation energies if, as was shown in Ref. 2 , one goes one step beyond the ASA and includes an interlayer monopole-dipole contribution.

Based on the observations above one is led to improve

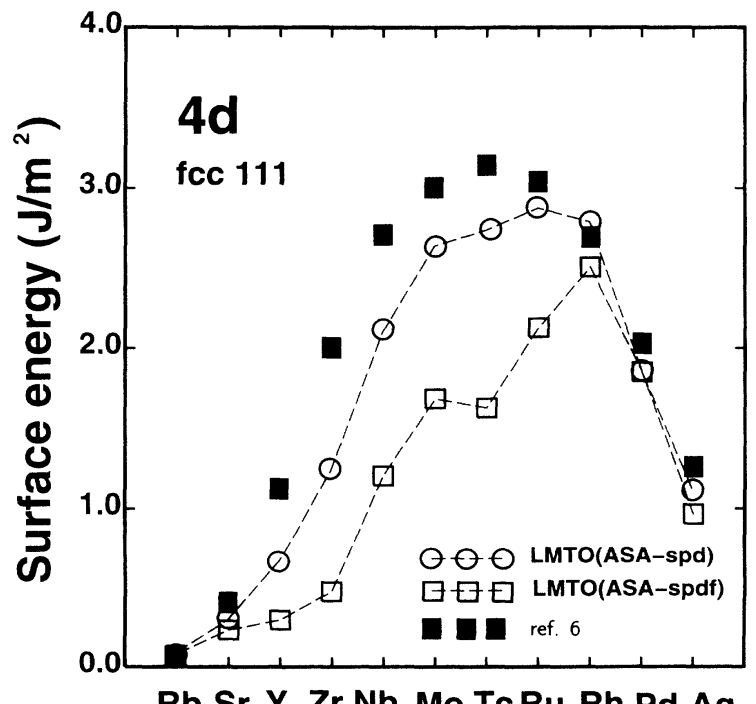

FIG. 1. Comparison of surface energies of the fcc (111) surface for the $4 d$ elements using $s, p, d$ and $s, p, d, f$ orbitals in an ASA calculation. The semiempirical values by de Boer et al. (Ref. 6) are also shown. 
the ASA total energy functional without sacrificing the efficiency of the Green's function technique. One possibility is to abandon the ASA completely and develop a localized Korringa-Kohn-Rostoker (KKR) scheme for surfaces, ${ }^{14}$ which will improve the description of the oneelectron energies. Here, we follow a different route where the idea is to retain the ASA as far as the potential and the calculation of the kinetic energy are concerned and use the spherically symmetric atomic potential to generate a complete, nonspherically symmetric charge density which in turn is applied in the evaluation of the remaining terms of the total energy. Such a procedure may be expected to work well because the kinetic energy is variational in the potential ${ }^{15}$ and because an accurate charge density may be obtained from an ASA potential. ${ }^{12}$ Furthermore, Svane and Andersen ${ }^{16}$ used essentially the approach proposed here in a successful calculation of fourcenter integrals and the cohesive energy of Si.

Apart from obtaining a more accurate description of the simple and transition metals, our aim is also to generalize the LMTO-Green's function method to treat real $f$-electron systems such as the light actinides. The results of the actinide calculations will be presented in a forthcoming publication. ${ }^{5}$ Here, we describe the full charge-density functional and present a series of calculations of the surface energies of the $4 d$ transition metals, the results of which are compared with the full-potential calculations. $^{1}$

\section{ENERGY FUNCTIONAL}

In this section we shall present a full charge-density functional (FCD), which is based on a spherically symmetric atomic-sphere potential and which preserves the important aspects of the ASA. Within the local density approximation $^{17}$ we define

$$
E_{\mathrm{FCD}}[n(\mathbf{r})]=\sum_{\mathbf{R}} T_{\mathrm{ASA}}^{R}+F[n(\mathbf{r})]
$$

where

$$
T_{\mathrm{ASA}}^{R}=\int^{E_{F}} \epsilon N^{R}(\epsilon) d \epsilon-\frac{1}{\sqrt{4 \pi}} \int_{S} n_{0}^{R, \mathrm{ASA}}(r) V_{\mathrm{eff}}^{R}(r) d^{3} r
$$

is the kinetic energy per site evaluated in the ASA and

$$
\begin{aligned}
F[n(\mathbf{r})]= & \int v_{\text {ext }}(\mathbf{r}) n(\mathbf{r}) d \mathbf{r}+\frac{1}{2} \iint \frac{n(\mathbf{r}) n\left(\mathbf{r}^{\prime}\right)}{\left|\mathbf{r}-\mathbf{r}^{\prime}\right|} d \mathbf{r} d \mathbf{r}^{\prime} \\
& +\int \epsilon_{\mathbf{x c}}[n(\mathbf{r})] n(\mathbf{r}) d \mathbf{r}
\end{aligned}
$$

In these equations $E_{F}$ is the Fermi level, $N^{R}(\epsilon)$ the site-projected one-electron state density, $S$ the atomic Wigner-Seitz radius, $n_{0}^{R, A S A}(r)$ the $l=0$ component of the electronic charge density at the site $\mathbf{R}, V_{\text {eff }}^{R}(r)$ the effective one-electron potential corresponding to the ASA energy functional, $v_{\text {ext }}(\mathbf{r})$ the potential from the nuclei, $n(\mathbf{r})$ the electronic charge density, and $\epsilon_{\mathbf{x c}}$ the exchangecorrelation energy density.

\section{A. Charge density}

Now the question arises how to construct an accurate, nonspherically symmetric crystal charge density which may be used to evaluate the functional (3). This problem was considered by Andersen et al., ${ }^{12}$ who used a multicenter expansion within the tight-binding LMTO formalism and showed that for silicon the charge density constructed from the output of an LMTO-ASA calculation was in excellent agreement with that obtained in a linear augmented plane-wave full-potential calculation. Furthermore, Svane and Andersen ${ }^{16}$ showed that the four-center integrals and the cohesive energy of silicon could be obtained with a high degree of accuracy from an ASA potential, and they attributed the remaining small deviation from other first-principles calculations of the cohesive energy to the use of the atomic sphere rather than the proper atomic cell in the evaluation of the total energy functional.

Here, we follow an even simpler procedure and construct the cell charge density by a one-center expansion rather than the multicenter expansion used by Andersen et al. ${ }^{12}$ However, we shall eventually evaluate the functional $F[n(\mathbf{r})]$ by integration over the proper atomic cells. The one-center expansion of the charge density in an ASA calculation may be written

$$
n^{\mathrm{ASA}}(\mathbf{r})=\sum_{L} n_{L}^{\mathrm{ASA}}(r) Y_{L}(\hat{\mathbf{r}})
$$

where $L$ is short-hand notation for $(l, m)$ and $Y_{L}$ is a real harmonic. In the ASA the charge density is normalized within the atomic sphere and, hence, generally only known in this region of space, but it may easily be calculated beyond the atomic radius. In the present case, we assume that the $L$ components of the charge density (4) are defined within the whole Wigner-Seitz $(W-S)$ cell, i.e., $0<r<S_{C}$, where $S_{C}$ is the radius of the sphere which circumscribes the $W-S$ cell. A realistic, nonoverlapping charge density may now be defined by

$$
n(\mathbf{r})=\left\{\begin{array}{l}
\sum_{L} n_{L}(r) Y_{L}(\hat{\mathbf{r}}) \text { for } \mathbf{r} \text { inside } W-S \text { cell } \\
0 \text { otherwise }
\end{array}\right.
$$

where

$$
n_{L}(r)=n_{L}^{\mathrm{ASA}}(r)+\delta_{l, 0} \Theta(r-S) a(1-S / r)^{2},
$$

$\Theta$ is the step function, and $\delta$ the Kronecker symbol. In (6) we have in the range $S<r<S_{C}$, i.e., at the outer corners of the $W-S$ cell, added a quadratic, oneparameter correction to the spherically symmetric part of the ASA charge density in order to ensure proper normalization of the charge density within the $W-S$ cell. By means of this simple functional form the charge density remains continuous and continuously differentiable at $S$ and the single parameter $a$ may be determined by the condition 


$$
\int_{W-S \text { cell }} n(\mathbf{r}) d \mathbf{r}=\int_{S} n^{\mathrm{ASA}}(\mathbf{r}) d \mathbf{r},
$$

which ensures that the $W-S$ cell contains the proper number of electrons.

\section{B. Cell integration}

To integrate within the $W-S$ cell we use the so-called shape function technique ${ }^{18,19}$ which was recently implemented by Drittler et $a .^{20}$ in their development of a full-potential Korringa-Kohn-Rostoker multiple scattering method. An alternative scheme to treat interstitial integrals has been proposed by Savrasov and Savrasov. ${ }^{21}$ Here, we apply the original version of the method and define the following shape function

$$
\sigma(\mathbf{r})=\left\{\begin{array}{l}
1 \text { for } \mathbf{r} \text { inside } W-S \text { cell } \\
0 \text { otherwise, }
\end{array}\right.
$$

which may be expanded in terms of real harmonics

$$
\sigma(\mathbf{r})=\sum_{L} \sigma_{L}(r) Y_{L}(\hat{\mathbf{r}}),
$$

where the partial component $\sigma_{L}(r)$ must be determined by numerical angular integration

$$
\sigma_{L}(r)=\int \sigma(\mathbf{r}) Y_{L}(\hat{\mathbf{r}}) d \Omega .
$$

In order to achieve the required accuracy, especially for larger $l$ values, the integration in (10) requires a careful consideration of the angular mesh points. In the present calculations we have chosen an angular mesh which reflects the symmetry of the $W-S$ cell.

By means of the shape function any integral over the $W-S$ cell may be transformed into an integral over the sphere which circumscribes the cell, i.e.,

$$
\int_{W-S \text { cell }} f(\mathbf{r}) d \mathbf{r}=\int_{S_{C}} \sigma(\mathbf{r}) f(\mathbf{r}) d \mathbf{r} .
$$

As a result, the charge density defined in (5) may be written as the expansion

$$
\begin{aligned}
n(\mathbf{r}) & =\sigma(\mathbf{r}) \sum_{L} n_{L}(r) Y_{L}(\hat{\mathbf{r}}) \\
& =\sum_{L} \tilde{n}_{L}(r) Y_{L}(\hat{\mathbf{r}}),
\end{aligned}
$$

where the partial radial functions

$$
\tilde{n}_{L}(r)=\sum_{L^{\prime}, L^{\prime \prime}} C_{L^{\prime}, L^{\prime \prime}}^{L} n_{L^{\prime}}(r) \sigma_{L^{\prime \prime}}(r)
$$

are given in terms of real-harmonic Gaunt coefficients $C_{L^{\prime}, L^{\prime \prime}}^{L}$ and the partial components of the shape function.

\section{Evaluation of $\boldsymbol{F}[\boldsymbol{n}(\mathbf{r})]$}

We shall now explain in detail how the different terms in the $F$ functional (3) may be calculated. If the total charge density is assumed to be the sum of nonoverlapping charge densities belonging to different cells as given in (5) or (12), the electrostatic part of the functional may be separated into intracell and intercell contributions. Furthermore, the total electrostatic and exchangecorrelation energy may be divided into contributions $F^{R}$ belonging to the cell at $\mathbf{R}$ and the functional evaluated as the sum over cells $F=\sum F^{R}$. For simplicity, we only give expressions for the contribution corresponding to the cell centered at the origin, i.e.,

$$
F^{0}=E_{\text {inter }}^{0}\left[\left\{Q_{L}^{R}\right\}\right]+E_{\text {intra }}^{0}\left[n^{0}\right]+E_{\text {xc }}^{0}\left[n^{0}\right],
$$

where we indicate that the intercell energy contribution depends on the multipole moments $Q_{L}^{R}$ of all other cells. The multipole moments of the charge density are defined as

$$
\begin{aligned}
Q_{L}^{R} & =\frac{(4 \pi)^{1 / 2}}{2 l+1} \int_{W-S \text { cell }}\left(\frac{r}{S}\right)^{l} n^{R}(\mathbf{r}) Y_{L}(\hat{\mathbf{r}}) d \mathbf{r} \\
& =\frac{(4 \pi)^{1 / 2}}{2 l+1} \int_{0}^{S_{C}}\left(\frac{r}{S}\right)^{l} \tilde{n}_{L}^{R}(r) r^{2} d r
\end{aligned}
$$

where we have kept the atomic radius $S$ in the expressions to make contact with the earlier ASA results.

The calculation of the electrostatic intercell energy contribution is a well-known problem and has been treated by many authors in different ways. ${ }^{19,22,23}$ Here, we follow the work of Gonis et al. ${ }^{22}$ in spite of the fact that their technique has problems in terms of $l$ convergence, which we shall return to in the next section. It has, however, the advantage of being formulated in terms of well-known quantities, such as multipole moments and LMTO structure constants.

If two cells are "far enough" apart their interaction energy may be given by the conventional Madelung energy of nonoverlapping charge distributions. However, for neighboring cells, i.e., for cells which are intersected by the bounding sphere of the cell at the origin, a correction term is needed. Thus, the intercell interaction energy belonging to the cell at the origin may be written

$$
\begin{aligned}
E_{\text {inter }}^{0}\left[\left\{Q_{L}^{R}\right\}\right]= & -\frac{1}{2 S} \sum_{L} Q_{L}^{0} \sum_{\mathbf{R}^{\prime}, L^{\prime}} S_{L, L^{\prime}}\left(\mathbf{R}^{\prime}\right) Q_{L^{\prime}}^{R^{\prime}} \\
& +\delta E_{\text {inter }}^{0}\left[\left\{Q_{L}^{R}\right\}\right] .
\end{aligned}
$$

Here, $S_{L, L^{\prime}}(\mathbf{R})$ are the conventional, i.e., unscreened, LMTO structure constants and the last term the "nearfield" corrections given by Gonis et al., ${ }^{22}$

$$
\begin{aligned}
\delta E_{\text {inter }}^{0}\left[\left\{Q_{L}^{R}\right\}\right]= & \frac{1}{2 S} \sum_{L} Q_{L}^{0} \sum_{\mathbf{R}_{n}, L^{\prime}} S_{L, L^{\prime}}\left(\mathbf{R}_{n}\right) Q_{L^{\prime}}^{R_{n}}-\frac{1}{2 S} \sum_{L} \sum_{\mathbf{R}_{n}} \frac{1}{2 l+1}\left(\frac{R_{n}}{S}\right)^{l} Y_{L}\left(\hat{\mathbf{R}_{n}}\right) \\
& \times \sum_{L^{\prime}, L^{\prime \prime}} Q_{L^{\prime}}^{0} \frac{4 \pi\left(2 l^{\prime \prime}-1\right) ! !}{(2 l-1) ! !\left(2 l^{\prime}-1\right) ! !} C_{L^{\prime}, L^{\prime \prime}}^{L} \delta_{l^{\prime \prime}, l+l^{\prime}} \sum_{L^{\prime \prime \prime}} S_{L^{\prime \prime}, L^{\prime \prime \prime}}\left(2 \mathbf{R}_{n}\right) Q_{L^{\prime \prime \prime}}^{R_{n}}
\end{aligned}
$$


rewritten in terms of real harmonics. In (17) $\mathbf{R}_{n}$ runs over the neighboring cells defined above.

The intracell energy may be determined by solving the $l$-dependent Poisson equation or by a numerical integration using the following formulas:

$$
\begin{aligned}
E_{\text {intra }}^{0}\left[n^{0}\right]= & \frac{(4 \pi)^{1 / 2}}{S} \sum_{L} \int_{0}^{S_{C}} \tilde{n}_{L}^{0}(r)\left[\left(\frac{r}{S}\right)^{l} P_{L}^{0}(r)\right. \\
& \left.+\left(\frac{r}{S}\right)^{-l-1} Q_{L}^{0}(r)\right] r^{2} d r
\end{aligned}
$$

where

$$
P_{L}^{0}(r)=\frac{(4 \pi)^{1 / 2}}{2 l+1} \int_{r}^{S_{C}} \tilde{n}_{L}^{0}\left(r^{\prime}\right)\left(\frac{r^{\prime}}{S}\right)^{-l-1}\left(r^{\prime}\right)^{2} d r^{\prime}
$$

and

$$
Q_{L}^{0}(r)=\frac{(4 \pi)^{1 / 2}}{2 l+1} \int_{0}^{r} \tilde{n}_{L}^{0}\left(r^{\prime}\right)\left(\frac{r^{\prime}}{S}\right)^{l}\left(r^{\prime}\right)^{2} d r^{\prime}
$$

Note that the multipole moments given in (15) may be expressed in terms of the function $Q_{L}^{0}(r)$ defined above as $Q_{L}^{0}=Q_{L}^{0}\left(S_{C}\right)$. Finally, the exchange-correlation energy of the cell at the origin may be expressed in the local density approximation as

$$
E_{\mathrm{xc}}^{0}\left[n^{0}\right]=\iint_{0}^{S_{C}} \epsilon_{\mathrm{xc}}\left[n^{0}(\mathbf{r})\right] n^{0}(\mathbf{r}) r^{2} d r d \Omega .
$$

\section{Spherical cell model}

To make contact with the earlier ASA description, using the new formalism discussed above, one may define a spherical cell model (SCM) in which the $W-S$ cells coincide with the atomic spheres of radius $S$. In that case, the shape function is given by

$$
\begin{aligned}
\sigma(\mathbf{r}) & =(4 \pi)^{-1 / 2} \sigma_{0}(r) \\
& = \begin{cases}1 & \text { for } r<S \\
0 & \text { otherwise }\end{cases}
\end{aligned}
$$

and $\tilde{n}_{L}(r)=n_{L}(r)=n_{L}^{\mathrm{ASA}}(r)$ for $r<S$. Furthermore, the integrations giving the multipole moments (15), the intracell energy (18), and the exchange-correlation energy (21) should be carried out over the atomic sphere of radius $S$. If we assume that the overlap between the spherical cells may be neglected, i.e., we ignore $\delta E_{\text {inter }}$ in the calculation of the intercell energy (16), the model obtained in this way describes an ASA calculation in which the nonspherically symmetric output charge density is used to evaluate the energy functional (1). Finally, the previously used ASA functional may be obtained if only the spherically symmetric charge density is retained and the higher multipole moments, except the monopoledipole Madelung contribution, are neglected.

\section{E. Convergence of the partial wave expansions}

There are two parameters in the formulation of the electrostatic energy terms given in the previous para- graphs which are crucial for the accuracy of the calculation: the cutoff value of the partial wave expansions of the shape function (9) and the cutoff value of the double $l$ sum in the correction (17). The latter was studied in detail by Gonis et al., ${ }^{22}$ and they found that the outer sum (over $L$ ) in (17) is convergent only if the inner sum (over $L^{\prime}$ and $L^{\prime \prime \prime}$ ) is already carried to convergency, and that without the correction $\delta E_{\text {inter }}$ no convergence could be achieved.

We have performed test calculations for an fcc lattice with a homogeneous charge density in the form of an evaluation of the electrostatic (Madelung) energy of the system. The results are shown in Fig. 2, where they are scaled so as to recover the Madelung constant, which equals 1.8 for nonoverlapping spherical cells, while the exact value for an fcc lattice is 1.79175 . In the calculations we assumed $l_{\max }^{\text {inner }}=l^{\text {outer }}+10$ in the $l$ summations in (17). First, it is seen, in agreement with the observation by Gonis et al. ${ }^{22}$ that no convergence is achieved without the correction, and second, that a satisfactory convergence is achieved for $l_{\max }=\mathbf{3 0}$ for the inner $l$ sum and $l_{\max }=20$ for the outer sum. These values have, therefore, been used in the surface energy calculations to be presented later. We note that the calculation of the near-field correction is very time consuming and that it typically contributes less than $2 \%$ to the surface energy, the reason being that only the difference between the correction in the bulk and at the surface contributes.

The partial wave expansion of the shape function (9) is strongly oscillatory and the convergence towards a step function is rather slow. However, the quantities derived from it by integration are quite well behaved, as may be seen in Fig. 3 where we show the intercell, intracell, and

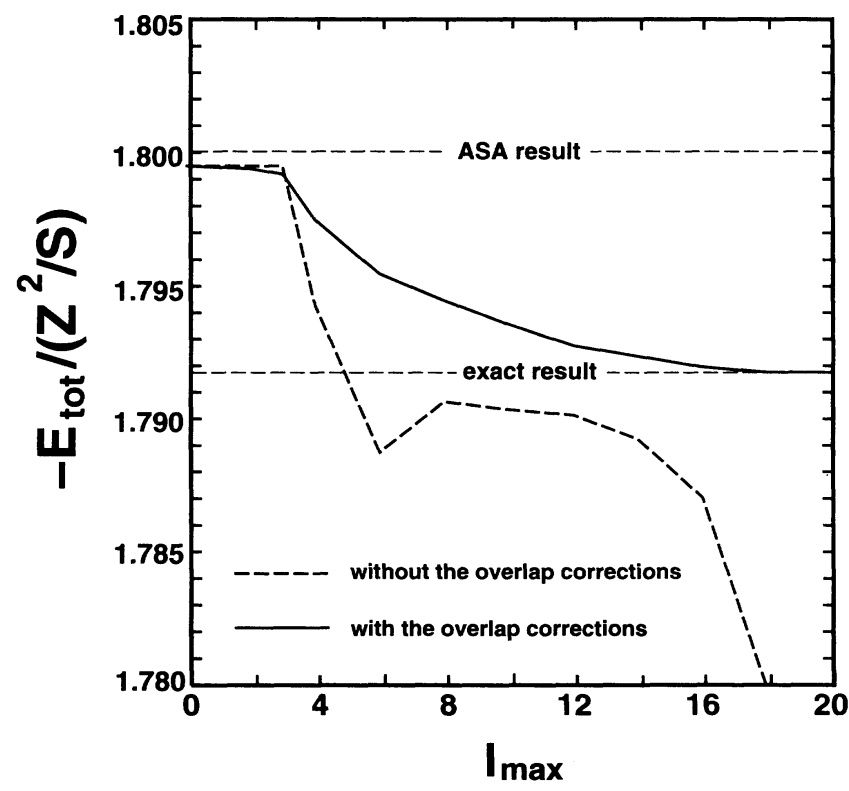

FIG. 2. Convergence test for the electrostatic energy of an fcc lattice with a homogeneous charge distribution using $(16,18)$ as a function of the maximal $l$ value used in the calculation. The total energy is scaled so as to yield the Madelung constant of the lattice ( $\alpha=1.79175$ for an fcc lattice). 


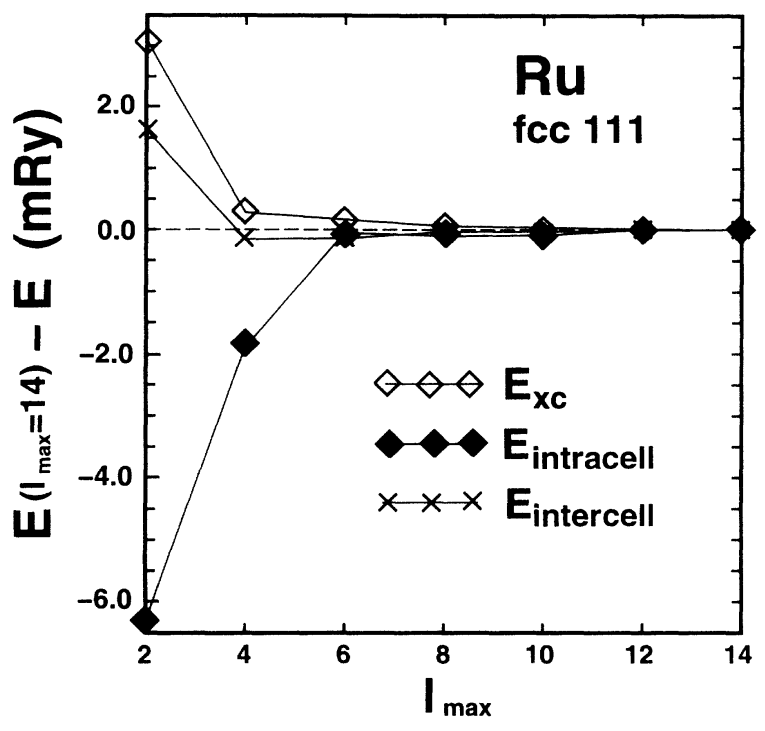

FIG. 3. Convergence test for the intercell, intracell, and exchange-correlation surface energy contributions in $\mathrm{Ru}$. The results are plotted relative to their converged values as a function of the maximal $l$ value used in the expansion (9) of the shape function.

exchange-correlation contributions to the surface energy of a $R u$ fcc (111) surface. Thus, a reasonable accuracy is achieved already for $l_{\max }=8-10$.

\section{RESULTS AND DISCUSSION}

With the formalism presented above we may now address the question of the accuracy of the ASA for surfaces and inter alia discuss the related problem of basis-set convergence of the LMTO. To do this, we show in Fig. 4 the $z$ components of the $p, d$, and $f$ multipole moments in the surface layer of the fcc (111) face of the $4 d$ elements. The moments are calculated by (15) assuming either a spherical cell model or a $W-S$ cell in which case one applies the shape function expansion (13). The figure shows that the spherical cell model reproduces the trends observed in the multipole moments, although the magnitude of the $d$ and $f$ moments is somewhat overestimated in this model.

In Fig. 4 we show for comparison the dipole moments obtained when the LMTO basis set is restricted to $s p d$ orbitals. It is seen that the main result of including $f$ orbitals in the basis set is to increase the dipole moment at the surface. This is to be expected, because the extra basis functions allow the charge density to become less spherically symmetric and thereby develop larger multipole moments. Since the ASA as used in the surface calculations ${ }^{2-4}$ is defined to include a negative interlayer monopole-dipole (Madelung) contribution to the total energy, the increase in the dipole moment upon inclusion of the $f$ orbitals is directly responsible for the low ASA-spdf surface energies shown in Fig. 1. On the other hand, it was exactly this inclusion of the monopole-

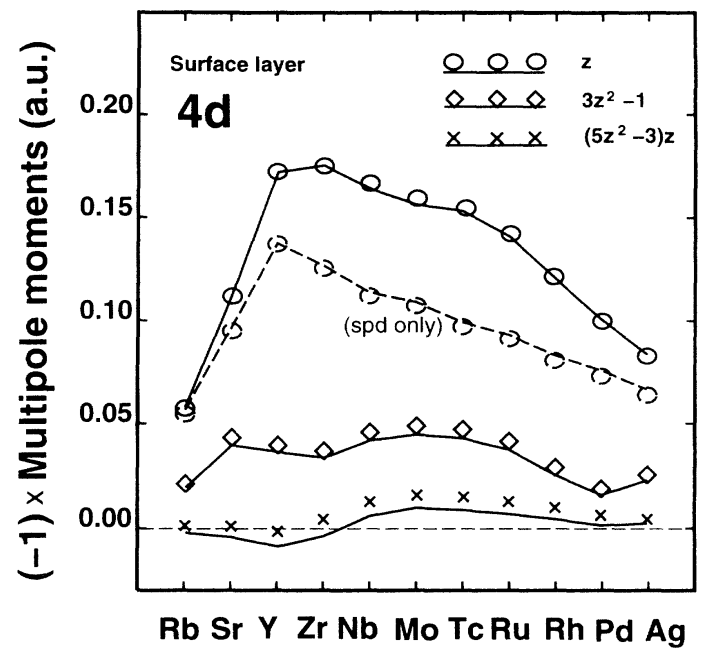

FIG. 4. The calculated $Q_{0, l}$ multipole moments for $p, d$, and $f$ states in the surface layer of the fcc (111) face of the metals $\mathrm{Rb}$ through Ag. The symbols correspond to the spherical cell results, while the results of the Wigner-Seitz integration are indicated by solid lines. For comparison we indicate by the broken line the dipole moment obtained in the spd calculations. The functional form of the relevant real harmonics is given at the top of the figure.

dipole energy (with the reduced dipole moment of an $s p d$ calculation) which was the reason for the excellent agreement between the ASA-spd results and the full-potential calculations. ${ }^{4}$ Thus, the inclusion of $f$ orbitals in the LMTO basis set destroys this agreement and calls for the functional described in Sec. II.

The functional $F[n(\mathbf{r})]$ may be partitioned according to (14) and in Fig. 5 we show those contributions to

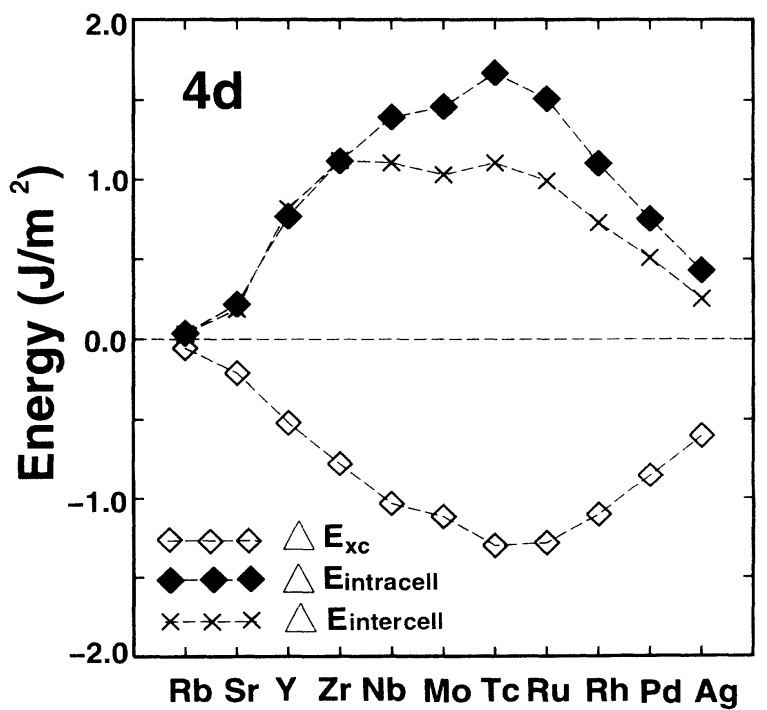

FIG. 5. Nonspherical contributions to the surface energy of the fcc (111) face of the $4 d$ series. We plotted the difference between the FCD and ASA results for the intercell and intracell Coulomb terms as well as the exchange-correlation energy. 
the intercell, intracell, and exchange-correlation energy which go beyond the ASA. For the intercell contribution this means all terms except the monopole-monopole (ss) and monopole-dipole $(s z)$ terms while for the two latter it means all terms except the $(s s)$ term. The contributions are calculated by means of the shape function technique and the correction (17) is included. As expected from the variational properties of the functional, there is a high degree of compensation between the intracell and the exchange-correlation energy and the correction to the ASA functional is, therefore, essentially that of the intercell Madelung contribution. Since this term is proportional to the products of multipole moments, among which the dipole moment provides the dominant contribution, the observed trend of the sum of the nonspherical energy contributions agrees with our expectation based on Fig. 4, i.e., it follows the square of the difference between $s p d$ and spdf dipole moments.

\section{A. Surface energy}

When all the nonspherically symmetric contributions to the functional are added to the (ASA-spdf) curve in Fig. 1 we obtain the surface energies shown in Fig. 6. The results labeled (FCD-spdf) in the figure and listed in Table I correspond to integration over the $W-S$ cell and include an spdf basis. They are expected to represent the best possible estimate of surface energies that may be obtained by means of a spherically symmetric atomic-sphere potential. It is seen in the figure that the $s p d$ and spdf results are close to each other as they should be for systems without occupied $f$ states. Since the individual contributions to the energy in the $s p d$ calculation are not completely converged in terms of the partial wave expansion this agreement is essentialy due to the separate variational properties of the kinetic energy and the $F[n(\mathbf{r})]$ functional. The surface energies in the SCM are somewhat larger than the results of the complete calculations and the difference seen in the figure is essentially a measure of the error caused by the integration over spheres rather than over the proper Wigner-Seitz cells.

The most rigorous test of the present calculation is the

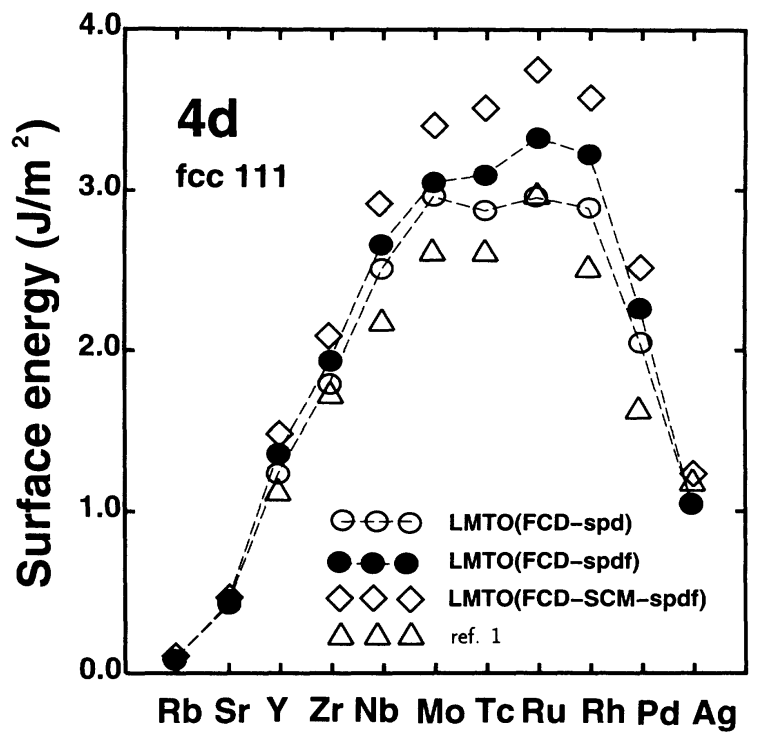

FIG. 6. Comparison of our final FCD surface energies for the fcc (111) surface for the $4 d$ elements using $s, p, d$ and $s, p, d, f$ states to the full potential slab calculation by Methfessel et al. (Ref. 1).

comparison with the full-potential results of Methfessel et $a{ }^{1}{ }^{1}$ In this test one should note that Methfessel et al. use a triple spd basis, and for that reason one should compare their surface energies with the (FCD-spd) results in Fig. 6. Furthermore, in contrast to the present Green's function technique, the full-potential calculations are nonrelativistic, relax all core states, include the effects of a $4 p$ semicore, and allow for a relaxation in the position of the surface layer. On the other hand, the Green's function technique treats the surface as a truly semi-infinite structure and does not rely on the slab supercell geometry used in the full-potential calculations. With these differences in numerical technique in mind it is gratifying to see that the agreement between the two sets of calculations is now such that the mean deviation in surface energy over the $4 d$ period has fallen from $18 \%$ to $10 \%$.

TABLE I. The atomic Wigner-Seitz radii $S_{\mathrm{ws}}$ corresponding to the experimentally obtained equilibrium lattice spacings, the calculated surface energy $\gamma$, and the calculated work function $W$ of the $4 d$ metals. The present theoretical results have been calculated by the full charge-density functional including spdf orbitals and integration over Wigner-Seitz cells by the shape function technique.

\begin{tabular}{lccccccccccc}
\hline \hline & $\mathrm{Rb}$ & $\mathrm{Sr}$ & $\mathrm{Y}$ & $\mathrm{Zr}$ & $\mathrm{Nb}$ & $\mathrm{Mo}$ & $\mathrm{Tc}$ & $\mathrm{Ru}$ & $\mathrm{Rh}$ & $\mathrm{Pd}$ & $\mathrm{Ag}$ \\
\hline$S_{\text {ws }}($ Bohr $)$ & 5.197 & 4.494 & 3.761 & 3.347 & 3.071 & 2.992 & 2.840 & 2.791 & 2.809 & 2.873 & 3.005 \\
$\gamma(\mathrm{eV} /$ atom $)$ & 0.13 & 0.43 & 0.96 & 1.08 & 1.25 & 1.36 & 1.23 & 1.29 & 1.27 & 0.93 & 0.47 \\
$\gamma\left(\mathrm{J} / \mathrm{m}^{2}\right)$ & 0.10 & 0.43 & 1.37 & 1.94 & 2.67 & 3.06 & 3.11 & 3.34 & 3.24 & 2.27 & 1.05 \\
\hline$W(\mathrm{eV})$ & 2.19 & 2.22 & 3.29 & 4.05 & 4.30 & 4.65 & 4.98 & 5.11 & 5.26 & 5.33 & 4.56 \\
$W^{\mathrm{a}}(\mathrm{eV})$ & & & 3.46 & 4.38 & 4.63 & 4.98 & 5.15 & 5.33 & 5.44 & 5.53 & 4.67 \\
$W^{\mathrm{b}}(\mathrm{eV})$ & 2.18 & 2.38 & 3.21 & 4.07 & 4.33 & 4.50 & 4.86 & 5.11 & 5.11 & 5.30 & 4.57 \\
\hline \hline
\end{tabular}

${ }^{a}$ Full potential LMTO calculations, Ref. 1.

${ }^{\text {b } L K K R ~ c a l c u l a t i o n s ~ u s i n g ~ s p d f ~} g$ orbitals, Ref. 24 . 


\section{B. Work function}

Within the LMTO-ASA Green's function technique the work function may be calculated as

$$
W=\Delta \phi-E_{F},
$$

where $E_{F}$ is the Fermi level of the bulk crystal with the ASA energy zero and $\Delta \phi$ the electrostatic barrier at the surface. Both quantities are affected by the size of the basis set and, in addition, $\Delta \phi$ is affected by the choice of functional. Since neither of the two obeys a variational principle one would expect an accurate estimate of $W$ to require absolute convergence in both terms. However, it turns out that a certain amount of cancellation takes place and as shown in Refs. 2-4 one may obtain excellent values for the work functions of metals even with the ASA functional and the conventional minimal spd basis.

In his basis-set convergence test of the ASA for the $5 d$ transition metals Crampin ${ }^{24}$ points to two sources of errors in the calculated work functions, (a) the determination of the Fermi level by integrating partial state densities and (b) the cutoff of the expansion of tails from neighboring sites. He finds that the combined effect in an $s p d$ calculation is to overestimate the Fermi level in the worst case, i.e., in the $5 d$ metal $\mathrm{Ir}$, by as much as $1.2 \mathrm{eV}$ and according to (23) this would lead to a low work function. The effect on the work function, however, is more than compensated by an underestimate of the magnitude of the dipole moment, cf. Fig. 4, which leads to an overestimate of the surface dipole since the dipole contribution to $\Delta \phi$ is negative. Crampin concludes that work function calculated by means of the usual minimal spd basis is overestimated by up to $0.7 \mathrm{eV}$ or $15 \%$ in the $5 d$ series.

In the present technique, the Fermi level is determined by a direct count of states in the calculated band structure and does not suffer from the errors introduced by integrating partial state densities. Therefore, the ASA results reported in Ref. 4 correspond to somewhat lower Fermi levels and slightly higher work functions than those of Crampin. ${ }^{24}$ In addition to the effect mentioned by Crampin, the cutoff in the tail expansion affects the charge density and hence the exchange-correlation potential $\mu_{\mathrm{xc}}(S)$ at the Wigner-Seitz sphere. As a result the entire band structure including the Fermi level is lowered when $f$ partial waves are included in the basis. To quantify, Crampin finds that the Fermi level in $\mathrm{Rh}, \mathrm{Pd}$, and $\mathrm{Ag}$ goes down by $0.70 \mathrm{eV}, 0.54 \mathrm{eV}$, and $0.45 \mathrm{eV}$, respectively, relative to $\mu_{\mathrm{xc}}(S)$ if $f$ states are included. The comparable shifts in the present calculation are $0.61 \mathrm{eV}, 0.38 \mathrm{eV}$, and $0.19 \mathrm{eV}$ to which one should add the corresponding changes of $0.30 \mathrm{eV}, 0.27 \mathrm{eV}$, and $0.21 \mathrm{eV}$, respectively, in the exchange-correlation potential at $S$ to find the effect on the calculated work function. Owing to the cancellation which takes place in (23) the net result is to make the work functions calculated by Crampin with an $s p d$ basis appear somewhat better converged than those reported in Ref. 4.

In Fig. 7 we show the work function of the fcc (111) face of the $4 d$ elements calculated at various levels of

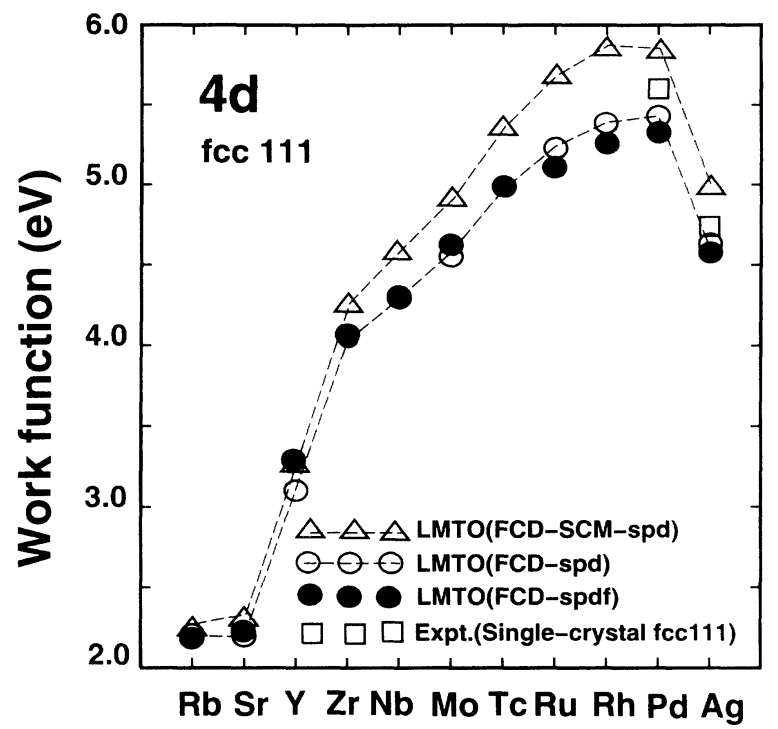

FIG. 7. Calculated work functions of the fcc (111) surface for the $4 d$ elements using $s, p, d$ and $s, p, d, f$ states. The available single-crystal experimental data are also shown.

approximation. In the comparison one notes that the spherical cell model, which corresponds to the previous ASA calculations, ${ }^{3,4}$ overestimates the work function by up to $0.5 \mathrm{eV}$. More importantly, however, one sees that when the integration over the proper Wigner-Seitz cell is included the results of the $s p d$ and the $s p d f$ calculations agree to better than $0.1 \mathrm{eV}$. The present work functions are in perfect agreement with the results obtained by Crampin $^{24}$ as may be seen in Table I. This is to be expected since the layered KKR technique ${ }^{24}$ is close to the present in its use of the ASA. The present results fall on the average $0.2 \mathrm{eV}$ below those obtained by Methfessel et al. ${ }^{1}$ which may be considered satisfactory in view of the differences in numerical technique.

\section{CONCLUSION}

We have studied the accuracy with which one may use the LMTO method in conjunction with a spherically symmetric potential to calculate surface properties of metals. In the study we use a functional where the kinetic energy is calculated completely in the ASA while the Coulomb and exchange-correlation energies are calculated on the basis of the nonspherically symmetric charge density generated within nonoverlapping, spacefilling Wigner-Seitz cells from the ASA potential. We find that although individual terms may not be completely converged the variational properties of the total energy functional and that of the kinetic energy lead to a surface energy of the fcc (111) face of the $4 d$ metals which is in close agreement with recent full-potential calculations. In addition, we find that the improved functional leads to a similar improvement in the calculated work functions. 


\section{ACKNOWLEDGMENTS}

We wish to thank Dr. S. Crampin for communicating his results prior to publication, for allowing us to quote his unpublished results for the $4 d$ transition metals, and for discussions concerning the convergence of the partial wave expansion. This work was supported in part by Grant No. ERB-CIPA-CT-92-2096 of the Commis- sion of the European Communities and research project OTKA 2950 of the Hungarian National Scientific Research Foundation. Center for Atomic-scale Materials Physics is sponsored by the Danish National Research Foundation. Part of the work was supported by grants from the Novo Nordisk Foundation and the Danish research councils through the Danish Center for Surface Reactivity.
${ }^{1}$ M. Methfessel, D. Hennig, and M. Scheffler, Phys. Rev. B 46, 4816 (1992).

${ }^{2}$ H. L. Skriver and N. M. Rosengaard, Phys. Rev. B 43, 9538 (1991).

${ }^{3}$ H. L. Skriver and N. M. Rosengaard, Phys. Rev. B 45, 9410 (1992).

${ }^{4}$ H. L. Skriver and N. M. Rosengaard, Phys. Rev. B 46, 7157 (1992).

${ }^{5}$ J. Kollár, L. Vitos, and H. L. Skriver, Phys. Rev. B 49, 11288 (1994).

${ }^{6}$ F. R. de Boer, R. Boom, W. C. M. Mattens, A. R. Miedema, and A. K. Niessen, Cohesion in Metals (NorthHolland, Amsterdam, 1988).

7 O. K. Andersen, Phys. Rev. B 12, 3060 (1975).

${ }^{8}$ H. L. Skriver, The LMTO Method (Springer-Verlag, Berlin, 1984).

${ }^{9}$ O. Gunnarsson, O. Jepsen, and O. K. Andersen, Phys. Rev. B 27, 7144 (1983).

${ }^{10}$ O. K. Andersen and O. Jepsen, Phys. Rev. Lett. 53, 2571 (1984).

11 O. K. Andersen, O. Jepsen, and D. Glötzel, in Highlights of Condensed-Matter Theory, edited by F. Bassani, F. Fumi, and M. P. Tosi (North-Holland, New York, 1985).

12 O. K. Andersen, Z. Pawlowska, and O. Jepsen, Phys. Rev. B 34, 5253 (1986).
${ }^{13}$ W. R. L. Lambrecht and O. K. Andersen, Surf. Sci. 178, 256 (1986); (private communication).

${ }^{14}$ L. Szunyogh, B. Ujfalussy, P. Weinberger, and J. Kollár, Phys. Rev. B 49, 2721 (1994)

${ }^{15}$ K. W. Jacobsen, J. K. Norskov, and M. J. Puska, Phys. Rev. B 35, 7423 (1987).

${ }^{16}$ A. Svane and O. K. Andersen, Phys. Rev. B 34, 5512 (1986).

${ }^{17}$ For the exchange-correlation potential and energy we use the Perdew-Zunger parametrization [J. Perdew and A. Zunger, Phys. Rev. B 23, 5048 (1981)], of the many-body calculations by Ceperley and Alder [D. M. Ceperley and B. J. Alder, Phys. Rev. Lett. 45, 566 (1980)].

${ }^{18}$ O. K. Andersen and R. G. Wooley, Mol. Phys. 26, 905 (1973).

19 J. van W. Morgan, J. Phys. C 10, 1181 (1977).

${ }^{20}$ B. Drittler, M. Weinert, R. Zeller, and P. H. Dederichs, Solid State Commun. 79, 31 (1991).

${ }^{21}$ S. Yu. Savrasov and D. Yu. Savrasov, Phys. Rev. B 46, 12181 (1992).

22 A. Gonis, E. C. Sowa, and P. A. Sterne, Phys. Rev. Lett. 66, 2207 (1991).

${ }^{23}$ G. H. Schadler, Phys. Rev. B 45, 11314 (1992).

${ }^{24} \mathrm{~S}$. Crampin (unpublished and private communication). 